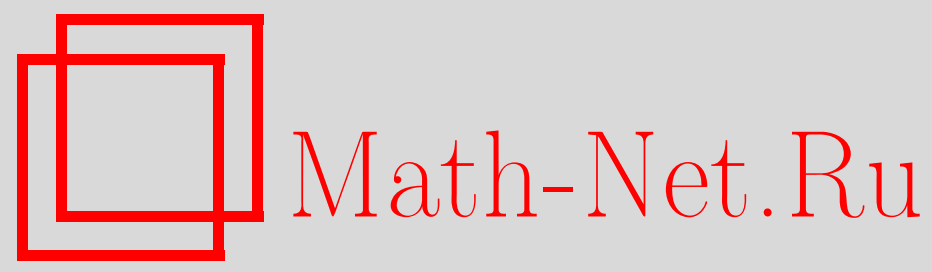

А. М. Бикчентаев, О минимальности топологии сходимости по мере на конечных алгебрах фон Неймана, Матем. заметки, 2004, том 75, выпуск 3, 342-349

DOI: https://doi.org/10.4213/mzm36

Использование Общероссийского математического портала Math-Net.Ru подразумевает, что вы прочитали и согласны с пользовательским соглашением http://www.mathnet.ru/rus/agreement

Параметры загрузки:

IP: 54.237 .59 .107

26 апреля 2023 г., $15: 18: 42$ 


\title{
О МИНИМАЛЬНОСТИ ТОПОЛОГИИ СХОДИМОСТИ ПО МЕРЕ НА КОНЕЧНЫХ АЛГЕБРАХ ФОН НЕЙМАНА
}

\section{А. М. Бикчентаев}

\begin{abstract}
Доказана непрерьвность естественного вложения метрического идеального пространства на конечной алгебре фон Неймана $\mathscr{M}$ в *-алгебру измеримых операторов $\widetilde{\mathscr{M}}$ с топологией сходимости по мере. С помошью этого факта установлено, что топология сходимости по мере является минимальной среди всех метризуемых топологий, согласованных со структурой кольца на $\widetilde{\mathscr{M}}$.

Библиографоия: 17 названий.
\end{abstract}

Пусть $(\Omega, \Sigma, \mu)$ - пространство с конечной мерой, $L^{0}(\mu)$ - алгебра всех $\mu$-измеримых функций на $\Omega$, снабженная топологией сходимости по мере. Непрерьвность естественного вложения классических функциональньх пространств в $L^{0}(\mu)$ играет важнейшую роль как в теории банаховых идеальных пространств, так и в теории интерполящии линейных операторов в этих пространствах. Бурное развитие теории некоммутативного интегрирования (см., например, [1]) привело к интенсивному изучению соответствующих аналогов идеальных функциональных пространств. В этой работе идеальные пространства измеримых операторов служат не только объектом исследования, но и выступают как инструмент (см. также [2]). Мы доказьваем непрерывность естественного вложения метрических идеальных пространств на алгебре фон Неймана $\mathscr{M}$ с точным нормальным конечным следом в *-алгебру $\widetilde{\mathscr{M}}$ измеримых операторов, снабженную топологией сходимости по мере. Любопытно, что этот факт позволяет получить основной результат заметки: топология сходимости по мере является минимальной среди всех метризуемых топологий, согласованных со структурой кольца на $\mathscr{\mathscr { M }}$. Метод доказательства является новьг даже для алгебры случайных величин $L^{0}(\mu)$.

1. Обозначения, определения и предварительные сведения. Пусть $G$ - коммутативная метризуемая топологическая группа в аддитивной записи, т.е. отображение $(x, y) \mapsto x-y$ декартова произведения $G \times G$ на $G$ непрерьвно. Метрика $\rho$ на групе $G$ назьвается инвариантной, если $\rho(a+x, a+y)=\rho(x, y)$ для всех $a, x, y \in G$. Положив $a=-y$, имеем эквивалентное условие $\rho(x-y, 0)=\rho(x, y)$ для всех $x, y \in G$ (поскольку $\rho(a+x, a+y)=\rho((a+x)-(a+y), 0)=\rho(x-y, 0)=\rho(x, y))$. Нам понадобится частный случай теоремы Какутани [3]: $T_{0}$-группа метризуема тогда и только тогда, когда

Работа выполнена при частичной поддержке Российского фонда фундаментальных исследований, грант № 01-01-00129, и научной программы "Университеты России - фундаментальные исследования", грант № УР.04.01.061. 
она имеет счетную открытую базу в 0 , причем метрика может быть выбрана инвариантной.

Множество $X \subset G$ назьвается ограниченным, если для каждой окрестности нуля $U$ найдется такое натуральное число $n$, что

$$
X \subset \underbrace{U+\cdots+U}_{n \text { раз }} .
$$

Для алгебры фон Неймана $\mathscr{M}$ операторов в гильбертовом пространстве через $\mathscr{M}^{\mathrm{pr}}$ будем обозначать ееподмножество проекторов; $e$ - единица алгебры $\mathscr{M}, \mathscr{M}_{1}$ - единичньй шар в $C^{*}$-норме $\|\cdot\|$ на $\mathscr{M}$. Пусть $\tau$ - точньй нормальный конечньй след на $\mathscr{M}$.

Множество $\widetilde{\mathscr{M}}$ всех замкнутьх плотно определенных присоединенных к $\mathscr{M}$ операторов замкнуто относительно перехода к сопряженному оператору, умножению на скаляр и операций сильного сложения и умножения, получаемых замыканием обычных операций. В *-алгебре $\widetilde{\mathscr{M}}$ вводится топология $t_{\tau}$ сходимости по мере [4], фундаментальную систему окрестностей нуля которой образуют множества

$$
U_{\varepsilon, \delta}=\left\{x \in \widetilde{\mathscr{M}}:\|x q\| \leqslant \varepsilon, \tau(e-q) \leqslant \delta \text { для некоторого } q \in \mathscr{M}^{\mathrm{pr}}\right\}, \quad \varepsilon>0, \quad \delta>0 .
$$

Известно, что $\left\langle\widetilde{\mathscr{M}}, t_{\tau}\right\rangle$ является полной метризуемой топологической $*$-алгеброй, причем $\mathscr{M}$ плотно в $\left\langle\mathscr{M}, t_{\tau}\right\rangle$.

Напомним [5], что подпространство $E \subset \widetilde{\mathscr{M}}$ с инвариантной метрикой $\rho$ назьвается метрическим идеальным пространством (далее МИП) на $\mathscr{M}$, если

1) $x \in E \Longrightarrow x^{*} \in E$ и $\rho(x, 0)=\rho\left(x^{*}, 0\right)$;

2) $x \in \widetilde{\mathscr{M}}, y \in E$ и $|x| \leqslant|y| \Longrightarrow x \in E$ и $\rho(x, 0) \leqslant \rho(y, 0)$,

где $|z|=\sqrt{z^{*} z}$ для $z \in \widetilde{\mathscr{M}}$. Среди МИП хорошо изучены некоммутативные $L^{p}$-пространства Лебега $(0<p<\infty)$ (см. [2], [6]), пространства Орлича [7], пространства Лоренца и пространства Марцинкевича [8], [9]. Для этих МИП непрерьвность естественного вложения в $\left\langle\widetilde{\mathscr{M}}, t_{\tau}\right\rangle$ была установлена с использованием специальных свойств соответствующих метрик. В [10] исследованы выпуклые $t_{\tau}$-ограниченные подмножества $\widetilde{\mathscr{M}}$ и изучены свойства оператора естественного вложения банаховых идеальных пространств на $\mathscr{M}$. Метрика

$$
\rho_{\tau}(x, y)=\inf _{q \in \mathscr{M}^{\mathrm{pr}}} \max \{\|(x-y) q\|, \tau(e-q)\}, \quad x, y \in \widetilde{\mathscr{M}}
$$

задает в $\widetilde{\mathscr{M}}$ структуру МИП с топологией сходимости по мере.

Напомним [11, с. 290], что $p, q \in \mathscr{M}^{\mathrm{pr}}$ назьваются әквивалентными (обозначение: $p \sim q$ ), если существует такой оператор $u \in \mathscr{M}$, что $p=u^{*} u$ и $q=u u^{*}$; запись $p \lesssim q$ означает, что $p \sim r$ для некоторого $r \leqslant q, r \in \mathscr{M}^{\mathrm{pr}}$. Через $s(x)$ будем обозначать левьй носитель оператора $x \in \widetilde{\mathscr{M}}$.

2. Основные результаты. Нам понадобятся следующиевспомогательные утверждения. 
Лемма 1. Для любой последовательности $\left\{p_{n}\right\} \subset \mathscr{M}^{\mathrm{pr}}$ существует такая попарно ортогональная последовательность $\left\{r_{n}\right\} \subset \mathscr{M}^{\mathrm{pr}}$, что $r_{n} \lesssim p_{n} \partial л я$ всех $n \in \mathbb{N}$ $u \bigvee_{n=1}^{\infty} p_{n}=\sum_{n=1}^{\infty} r_{n}$.

ДокАЗАТЕЛЬСТво. Методом математической индукции установим утверждение для конечных наборов проекторов. Для набора из двух проекторов $\{p, q\}$ положим $r_{1}=p$. Тогда $r_{2}=p \vee q-p \sim q-p \wedge q \leqslant q$, т.е. набор $\left\{r_{1}, r_{2}\right\}$ - искомьй. Пусть утверждение леммы верно для набора $\left\{p_{1}, \ldots, p_{k}\right\}$, т.е. $\bigvee_{n=1}^{k} p_{n}=\sum_{n=1}^{k} r_{n}, r_{n} \lesssim p_{n}, r_{n} r_{i}=0$ $(n \neq i), i, n \in\{1, \ldots, k\}$. Для набора $\left\{p_{1}, \ldots, p_{k+1}\right\}$ положим $q=\bigvee_{n=1}^{k} p_{n} ;$ тогда

$$
\bigvee_{n=1}^{k+1} p_{n}=\left(\bigvee_{n=1}^{k+1} p_{n}-q\right)+q=r_{k+1}+\sum_{n=1}^{k} r_{n}=\sum_{n=1}^{k+1} r_{n}
$$

Имеем

a) $r_{k+1} q=0 \Longrightarrow r_{k+1} r_{n}=0, n \in\{1, \ldots, k\}$;

б) $r_{k+1}=\bigvee_{n=1}^{k+1} p_{n}-q=p_{k+1} \vee q-q \sim p_{k+1}-p_{k+1} \wedge q \leqslant p_{k+1}$,

т.е. набор $\left\{r_{1}, \ldots, r_{k+1}\right\}$ искомый. Применив теорему Вижье, имеем

$$
\bigvee_{n=1}^{\infty} p_{n}=\lim _{k \rightarrow \infty} \bigvee_{n=1}^{k} p_{n}=\lim _{k \rightarrow \infty} \sum_{n=1}^{k} r_{n}=\sum_{n=1}^{\infty} r_{n}
$$

где пределы понимаются в смысле сильной операторной топологии. Лемма доказана.

Лемма 2. Пусть $\langle E, \rho\rangle-$ МИП на $\mathscr{M}$. Если $x \in \widetilde{\mathscr{M}} u x^{*} x \in E$, mo $x x^{*} \in E u$ $\rho\left(x^{*} x, 0\right)=\rho\left(x x^{*}, 0\right)$. В частности, если $p, q \in \mathscr{M}^{\mathrm{pr}}, p \lesssim q u q \in E$, mо $p \in E u$ $\rho(p, 0) \leqslant \rho(q, 0)$.

ДокАЗАТЕЛЬСтво. Пусть $z \in E$ и $u, v \in \mathscr{M}_{1}$. Тогда $|u z|^{2}=z^{*} u^{*} u z \leqslant z^{*} z=|z|^{2}$, и в силу операторной монотонности функции $\lambda \mapsto \sqrt{\lambda}(0 \leqslant \lambda<\infty)$ имеем $|u z| \leqslant|z|$. По свойству 2) МИП оператор $u z \in E$ и $\rho(u z, 0) \leqslant \rho(z, 0)$. По свойству 1$)$ МИП, переходя к сопряженным операторам, аналогично получаем $z v \in E$ и $\rho(z v, 0) \leqslant \rho(z, 0)$. Если $x=u|x|, x^{*}=v\left|x^{*}\right|$-полярные разложения операторов $x, x^{*} \in \widetilde{\mathscr{M}}$, то $x x^{*}=u|x|^{2} u^{*}=$ $u x^{*} x u^{*}, x^{*} x=v\left|x^{*}\right|^{2} v^{*}=v x x^{*} v^{*}$. Остальное теперь очевидно.

Лемма 3. Пусть $\langle E, \rho\rangle-$ МИП на $\mathscr{M} u\left\{x_{n}\right\} \subset E$. Тогда

$$
\rho\left(x_{n}, 0\right) \rightarrow 0 \quad(n \rightarrow \infty) \Longleftrightarrow \forall \lambda \in \mathbb{C} \quad\left(\rho\left(\lambda x_{n}, 0\right) \rightarrow 0, \quad n \rightarrow \infty\right)
$$

ДокАЗАТЕЛЬСТво. Пусть $\lambda \in \mathbb{C}$ и $k$-целая часть числа $|\lambda|+1$. Применяя свойство 2) МИП, неравенство треугольника и метод математической индукции, имеем оценку

$$
\rho\left(\lambda x_{n}, 0\right) \leqslant \rho\left(k x_{n}, 0\right) \leqslant k \rho\left(x_{n}, 0\right) \rightarrow 0, \quad n \rightarrow \infty .
$$

Следующее некоммутативное обобщение теоремы П. Щептыцкого [12] фактически было установлено в [5]. 
Теорема 1. Пусть $\mathscr{M}$ - алгебра фон Неймана с точныц нормальным конечным следом $\tau$ и $\langle Е, \rho\rangle-$ МИП на М. Тогда

I) если $x_{n}, x \in E$ и $\rho\left(x_{n}, x\right) \rightarrow 0$, mо $x_{n} \rightarrow x$ по мере;

II) если $\left\{x_{n}\right\}$ - последовательность Коши в $\langle E, \rho\rangle$, то существует $x \in \widetilde{\mathscr{M}}$, для которого $x_{n} \rightarrow x$ по мере;

III) если $X \subset \widetilde{\mathscr{M}}$ ограничено в $\langle E, \rho\rangle$, то оно ограничено в $\left\langle\widetilde{\mathscr{M}}, \rho_{\tau}\right\rangle$.

ДокАЗАТЕЛЬСТво. I) Будем считать $x=0$ и для определенной по формуле (1) метрики $\rho_{\tau}$ воспользуемся ее новым выражением из $[6$, c. 94$]$ :

$$
\rho_{\tau}(z, 0)=\inf _{\lambda>0} \max \left\{\lambda, \tau\left(e_{(\lambda, \infty)}(|z|)\right)\right\}, \quad z \in \widetilde{\mathscr{M}} .
$$

Здесь $e_{(\lambda, \infty)}(|z|)$ - спектральньй проектор оператора $|z|$, соответствующий интервалу $(\lambda, \infty)$. Если утверждение не верно, то существует такая последовательность операторов $\left\{x_{n}\right\} \subset E$ и число $\delta>0$, что $\rho\left(x_{n}, 0\right) \rightarrow 0$ при $n \rightarrow \infty$, но $\rho_{\tau}\left(x_{n}, 0\right) \geqslant \delta$ при всех $n \in \mathbb{N}$. Используя лемму 3 и переходя при необходимости к подпоследовательности, считаем $\left\{x_{n}\right\} \subset E$ и число $\lambda>0$ такими, что $\rho\left(\lambda^{-1} x_{n}, 0\right)<2^{-n}$ и для $p_{n}=e_{(\lambda, \infty)}\left(\left|x_{n}\right|\right)$ выполнено неравенство $\tau\left(p_{n}\right) \geqslant 2 \lambda$ при всех $n \in \mathbb{N}$. Пусть $q_{n}=\bigvee_{k \geqslant n} p_{k} ;$ тогда $q_{n} \searrow$ и $\tau\left(q_{n}\right) \geqslant 2 \lambda$. Для $q=\bigwedge_{n \geqslant 1} q_{n}$ в силу нормальности следа $\tau$ имеем $\inf _{n} \geqslant 1 \tau\left(q_{n}\right) \geqslant 2 \lambda$. Покажем возможность выбора натуральных чисел $k_{n} \geqslant n$ с условием $\tau(p) \geqslant \lambda$ для $p=\bigwedge_{n \geqslant 1} q_{n, k_{n}}$, где $q_{n, k_{n}}=\bigvee_{k=n}^{k_{n}} p_{k}$. Тогда $p \leqslant q$ и

$$
q-p=q-\left(\bigwedge_{n \geqslant 1} q_{n, k_{n}}\right) \wedge q=q-\bigwedge_{n \geqslant 1}\left(q \wedge q_{n, k_{n}}\right)=\bigvee_{n \geqslant 1}\left(q-q \wedge q_{n, k_{n}}\right)
$$

По лемме 1 и в силу нормальности следа $\tau$ имеем $\tau(q-p) \leqslant \sum_{n \geqslant 1} \tau\left(q-q \bigwedge q_{n, k_{n}}\right)$. Проекторы конечной алгебры $\mathscr{M}$ образуют непрерывную геометрию [13, теорема 6.5], т.е. полную модулярную решетку с дополнениями, удовлетворяющую для любой возрастающей сети $\left\{r_{\alpha}\right\}_{\alpha \in A} \subset \mathscr{M}^{\mathrm{pr}}$ и для любого $r \in \mathscr{M}^{\mathrm{pr}}$ условию

$$
r \wedge\left(\bigvee_{\alpha \in A} r_{\alpha}\right)=\bigvee_{\alpha \in A}\left(r \wedge r_{\alpha}\right)
$$

и дуальному ему условию. Поэтому при $k_{n} \rightarrow \infty$ получаем

$$
q_{n, k_{n}} \wedge q \nearrow q_{n} \wedge q=q
$$

Для каждого $n \in \mathbb{N}$ найдется такое $k_{n} \in \mathbb{N}$, что $\tau\left(q-q \wedge q_{n, k_{n}}\right)<2^{-n} \lambda$. Теперь для $p=$ $\bigwedge_{n \geqslant 1} \bigvee_{k=n}^{k_{n}} p_{k}$ имеем $\tau(p) \geqslant \lambda$. Неравенство $\lambda p_{n} \leqslant\left|x_{n}\right|$ показывает, что $\rho\left(p_{n}, 0\right) \leqslant 2^{-n}$ для всех $n \in \mathbb{N}$. Используя (см. лемму 1) представление $\bigvee_{k=n}^{k_{n}} p_{k}=\sum_{k=n}^{k_{n}} r_{k}$, лемму 2 и неравенство треугольника для метрики $\rho$, устанавливаем, что проектор $\bigvee_{k=n}^{k_{n}} p_{k}$, а в силу свойства 2) МИП вместе с ним и проектор $p$ лежат в каждом $\varepsilon$-шаре метрики $\rho$. Следовательно, $p=0$ в $\langle E, \rho\rangle$ и в силу алгебраичности включения $E \subset \widetilde{\mathscr{M}}$ имеем $p=0 \in \widetilde{\mathscr{M}}$ - противоречие. 
II) В силу полноты МИП $\left\langle\widetilde{\mathscr{M}}, \rho_{\tau}\right\rangle$ достаточно проверить фундаментальность последовательности $\left\{x_{n}\right\}$ по мере. Если это не так, то существует такое $\varepsilon>0$, что для любого $n \in \mathbb{N}$ найдутся $i_{n} \geqslant j_{n} \geqslant n$, что $\rho_{\tau}\left(x_{i_{n}}-x_{j_{n}}, 0\right) \geqslant \varepsilon$. Тогда $i_{n}-j_{n} \rightarrow \infty, n \rightarrow \infty$, и $\rho\left(x_{i_{n}}-x_{j_{n}}, 0\right) \rightarrow 0, n \rightarrow \infty$, откуда в силу п. I) имеем $\rho_{\tau}\left(x_{i_{n}}-x_{j_{n}}, 0\right) \rightarrow 0, n \rightarrow \infty,-$ противоречие.

III) По п. I) тождественное вложение $E$ в $\widetilde{\mathscr{M}}$ непрерьвно, а любое непрерывное отображение переводит ограниченные множества в ограниченные. Теорема доказана.

С помощью теоремы 1 получим новое доказательство следующего известного факта.

СлЕДСТВИЕ 1. Для алгебры фон Неймана $\mathscr{M}$ с точным нормальным конечным следом топология сходимости по мере на $\widetilde{\mathscr{M}}$ не зависит от выбора такого следа.

ДокАЗАТЕЛЬСТво. Пусть $\tau$ и $\mu$-два точных нормальных конечных следа на $\mathscr{M}$. По п. І) теоремы МИП $\left\langle\widetilde{\mathscr{M}}, \rho_{\mu}\right\rangle$ непрерывно вложено в $\left\langle\widetilde{\mathscr{M}}, t_{\tau}\right\rangle$; аналогично, МИП $\left.\widetilde{\mathscr{M}}, \rho_{\tau}\right\rangle$ непрерьвно вложено в $\left\langle\widetilde{\mathscr{M}}, t_{\mu}\right\rangle$. Следовательно, $t_{\tau}=t_{\mu}$.

Рассмотрим еще один пример МИП на алгебре фон Неймана $\mathscr{M}$ с точным нормальным конечным следом $\tau$. Пусть $\rho_{\mathrm{s}, \tau}(x, y)=\tau(\mathrm{s}(x-y))$ для всех $x, y \in \widetilde{\mathscr{M}}$. Поскольку из соотношений $\mathrm{s}(x+y)) \leqslant \mathrm{s}(x) \vee \mathrm{s}(y), \mathrm{s}(x y) \leqslant \mathrm{s}(x), \mathrm{s}(x y) \lesssim \mathrm{s}(y)$ вытекают оценки

$$
\begin{aligned}
\rho_{\mathrm{s}, \tau}(x+y, 0) & =\tau(\mathrm{s}(x+y)) \leqslant \tau(\mathrm{s}(x) \vee \mathrm{s}(y)) \leqslant \tau(\mathrm{s}(x))+\tau(\mathrm{s}(y))=\rho_{\mathrm{s}, \tau}(x, 0)+\rho_{\mathrm{s}, \tau}(y, 0), \\
\rho_{\mathrm{s}, \tau}(x y, 0) & =\tau(\mathrm{s}(x y)) \leqslant \min \{\tau(\mathrm{s}(x)), \tau(\mathrm{s}(y))\}=\min \left\{\rho_{\mathrm{s}, \tau}(x, 0), \rho_{\mathrm{s}, \tau}(y, 0)\right\}
\end{aligned}
$$

для всех $x, y \in \widetilde{\mathscr{M}}$, инвариантная метрика $\rho_{\mathrm{s}, \tau}$ задает согласованную со структурой кольца на $\mathscr{M}$ топологию $t_{\mathrm{s}, \tau}$. Очевидно, $\rho_{\tau}(x, 0) \leqslant \rho_{\mathrm{s}, \tau}(x, 0)$ для всех $x \in \mathscr{M}$. Произведение $(\lambda, x) \mapsto \lambda x\left(\langle\mathbb{C},|\cdot|\rangle \times\left\langle\widetilde{\mathscr{M}}, \rho_{\mathrm{s}, \tau}\right\rangle \rightarrow\left\langle\widetilde{\mathscr{M}}, \rho_{\mathrm{s}, \tau}\right\rangle\right)$ не является непрерьвным: для последовательности $x_{n}=n^{-1}$ п при больших $n \in \mathbb{N}$ имеем

$$
n^{-1}=\rho_{\tau}\left(x_{n}, 0\right)<\rho_{\mathrm{s}, \tau}\left(x_{n}, 0\right)=\tau(e)=\text { const. }
$$

Тем самьм $t_{\tau} \underset{\neq}{\subset} t_{\mathrm{s}, \tau}$. Поскольку $\mathrm{s}\left(x^{*}\right) \sim \mathrm{s}(x)$, имеем $\rho_{\mathrm{s}, \tau}\left(x^{*}\right)=\rho_{\mathrm{s}, \tau}(x)$, т.е. инволюция $x \mapsto x^{*}\left(x \in \stackrel{\mathcal{\mathscr { M }}}{)} t_{\mathrm{s}, \tau}\right.$-непрерывна. Отметим, что МИП $\left.\widetilde{\mathscr{M}}, \rho_{\mathrm{s}, \tau}\right\rangle$ играет важную роль в теории интерполяционных пространств.

Следующее утверждение является некоммутативньм обобщением основного результата работы Н. Т. Пека [14].

Теорема 2. Пусть $\mathscr{M}$ - алгебра фон Неймана с точным нормальным конечным следом $\tau$. Топология сходимости по мере является минимальной среди всех метризуемых топологий, согласованных со структурой кольиа на $\widetilde{\mathscr{M}}$.

ДокАЗАТЕльство. Пусть метризуемая согласованная со структурой кольца на $\widetilde{\mathscr{M}}$ топология $t \subset t_{\tau}$. По теореме Какутани топология $t$ задается инвариантной метрикой $\rho$. Взяв при необходимости топологически эквивалентную инвариантную метрику $\rho(x, y) /(1+\rho(x, y))$, считаем $\sup _{x, y \in \widetilde{\mathscr{M}}} \rho(x, y) \leqslant 1$. Покажем, что метрическое пространство $\langle\widetilde{\mathscr{M}}, \rho\rangle$ допускает топологически эквивалентную “идеальную” перенормировкy

$$
\tilde{\rho}(x, 0)=\sup _{a, b \in \mathscr{M}_{1}} \rho(a x b, 0), \quad x \in \widetilde{\mathscr{M}}
$$


Проверим аксиомы МИП для $\langle\widetilde{\mathscr{M}}, \tilde{\rho}\rangle$. Поскольку $\tilde{\rho}(x, 0) \geqslant \rho(x, 0)$ для всех $x \in \widetilde{\mathscr{M}}$, имеем $\tilde{\rho}(x, 0)=0 \Longleftrightarrow x=0$. Известно, что для конечной алгебры фон Неймана каждый оператор $x \in \widetilde{\mathscr{M}}$ может быть представлен в виде $x=u|x|$ с унитарным оператором $u \in \mathscr{M}$.

Действительно, пусть $x=v|x|$ - полярное разложение с частичной изометрией $v \in$ $\mathscr{M}$ и пусть $v^{*} v=f, v v^{*}=g$. Поскольку алгебра $\mathscr{M}$ конечна, проекторы $e-f$ и $e-g$ также эквивалентны. Пусть $e-f=w^{*} w$ и $e-g=w w^{*}$ с частичной изометрией $w \in \mathscr{M}$. Тогда оператор $u=v+w$ удовлетворяет условию $x=u|x|$ и является изометрией. Так

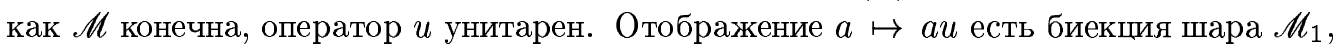
поэтому

$$
\tilde{\rho}(x, 0)=\sup _{a, b \in \mathscr{M}_{1}} \rho(a u|x| b, 0)=\sup _{c, b \in \mathscr{M}_{1}} \rho(c|x| b, 0)=\tilde{\rho}(|x|, 0), \quad x \in \widetilde{\mathscr{M}} .
$$

Если $x, y \in \widetilde{\mathscr{M}}_{\text {и }} 0 \leqslant x \leqslant y$, то существует такой оператор $z \in \mathscr{M}_{1}$, что $x=z y z^{*}[15$, c. 261], поэтому

$$
\tilde{\rho}(x, 0)=\sup _{a, b \in \mathscr{M}_{1}} \rho\left(a z y z^{*} b, 0\right) \leqslant \tilde{\rho}(y, 0) .
$$

Итак, если $|x| \leqslant|y|$, то $\tilde{\rho}(x, 0) \leqslant \tilde{\rho}(y, 0)$. Очевидно, что $\tilde{\rho}(x, y) \leqslant \tilde{\rho}(x, z)+\tilde{\rho}(z, y)$ для всех $x, y, z \in \mathscr{M}$. Отображение $b \mapsto u^{*} b$ есть биекция шара $\mathscr{M}_{1}$, поэтому для $x \in \widetilde{\mathscr{M}}$ имеем

$$
\tilde{\rho}\left(x^{*}, 0\right)=\sup _{a, b \in \mathscr{M}_{1}} \rho\left(a x^{*} b, 0\right)=\sup _{a, b \in \mathscr{M}_{1}} \rho\left(a|x| u^{*} b, 0\right)=\sup _{a, c \in \mathscr{M}_{1}} \rho(a|x| c, 0)=\tilde{\rho}(x, 0)
$$

по вышедоказанному. Покажем, что

$$
\rho\left(x_{n}, 0\right) \rightarrow 0 \quad(n \rightarrow \infty) \Longleftrightarrow \tilde{\rho}\left(x_{n}, 0\right) \rightarrow 0, \quad n \rightarrow \infty,
$$

для всех последовательностей $\left\{x_{n}\right\} \subset \widetilde{\mathscr{M}}$. Поскольку $\rho \leqslant \tilde{\rho}$, импликация ( $\Longleftarrow$ вьполнена. Если импликация $(\Longrightarrow)$ неверна, переходя при необходимости к подпоследовательности считаем, что для $\left\{x_{n}\right\} \subset \widetilde{\mathscr{M}}$ выполнено условие $\rho\left(x_{n}, 0\right) \rightarrow 0(n \rightarrow \infty)$ и

$$
\exists \delta>0, \quad \exists\left\{a_{n}\right\},\left\{b_{n}\right\} \subset \mathscr{M}_{1} \quad \forall n \in \mathbb{N}\left(\rho\left(a_{n} x_{n} b_{n}, 0\right) \geqslant \delta>0\right) .
$$

Заметим, что

$$
\rho_{\tau}(x, 0)=\inf _{q \in \mathscr{M}^{\mathrm{pr}}} \max \{\|x q\|, \tau(e-q)\} \leqslant\|x\|, \quad x \in \mathscr{M} .
$$

Поэтому последовательности операторов $\left\{a_{n}\right\}$ и $\left\{b_{n}\right\}$, будучи ограниченными в $C^{*}$-норме алгебры $\mathscr{M}$, являются $t_{\tau}$-ограниченньми (это следует и из п. III) теоремы 1 для МИП $\langle\mathscr{M},\|\cdot\|\rangle)$. Поскольку $t \subset t_{\tau}$, они и $t$-ограничены. Так как топология $t$ согласована со структурой кольца на $\mathscr{M}$, произведение (слева и справа) $t$-ограниченных последовательностей $\left\{a_{n}\right\}$ и $\left\{b_{n}\right\}$ на $t$-сходящуюся к нулю последовательность $\left\{x_{n}\right\}$ снова $t$-сходится к нулю - противоречие с (2).

Итак, $\langle\widetilde{\mathcal{M}}, \tilde{\rho}\rangle$ есть МИП на $\mathscr{M}$. Применив к $\langle\widetilde{\mathscr{M}}, \tilde{\rho}\rangle$ п. I) теоремы 1 , имеем $t_{\tau} \subset t$. Тем самым $t=t_{\tau}$. Теорема доказана. 
ЗАмЕчАниЕ 1. В формулировке теоремы 2 не требуется непрерывности инволюции $x \mapsto x^{*}, x \in \widetilde{\mathscr{M}}$.

ЗАмечАниЕ 2. Для коммутативной алгебры фон Неймана условие “идеальности" метрики в теореме 1 (см. [16]), как и условие согласованности топологии со структурой кольца в теореме 2 нельзя опустить (см. [14, теорема 1.1]). Доказательство теоремы 2 с помощью теоремы 1 является новым и в коммутативном случае.

В [17] введена топология $d_{\tau}$ двусторонней сходимости по мере на алгебре $\widetilde{\mathscr{M}}$, фундаментальную систему окрестностей нуля которой образуют множества

$$
V_{\varepsilon, \delta}=\left\{x \in \widetilde{\mathscr{M}}:\|q x q\| \leqslant \varepsilon, \tau(e-q) \leqslant \delta \text { для некоторого } q \in \mathscr{M}^{\mathrm{pr}}\right\}, \quad \varepsilon>0, \quad \delta>0 .
$$

Лемма 4. Отображения $(x, y) \mapsto x+y u(x, y) \mapsto x y(\widetilde{\mathscr{M}} \times \widetilde{\mathscr{M}} \rightarrow \widetilde{\mathscr{M}})$ непрерывны в топологии $d_{\tau}$.

ДокАЗАтЕльство. Покажем включения $V_{\varepsilon, \delta}+V_{\alpha, \beta} \subset V_{\varepsilon+\alpha, \delta+\beta}$ и $V_{\varepsilon, \delta} \cdot V_{\alpha, \beta} \subset$ $V_{\varepsilon \alpha, 2 \delta+2 \beta}$ для всех $\varepsilon, \delta, \alpha, \beta>0$. Пусть операторы $x \in V_{\varepsilon, \delta}$ и $y \in V_{\alpha, \beta}$ произвольны. Существуют такие $p, q \in \mathscr{M}^{\mathrm{pr}}$, что $\|p x p\| \leqslant \varepsilon,\|q y q\| \leqslant \alpha$ и $\tau(e-p) \leqslant \delta, \tau(e-$ $q) \leqslant \beta$. Для проектора $f=p \wedge q$ имеем $\tau(e-f)=\tau((e-p) \vee(e-q)) \leqslant \delta+\beta$; равенство $f(x+y) f=f(p x p+q y q) f$ влечет оценку $\|f(x+y) f\| \leqslant \varepsilon+\alpha$. Следуя [4], определим для произвольных операторов $z \in \widetilde{\mathscr{M}}_{\text {и }} v \in \mathscr{M}^{\mathrm{pr}}$ проектор $v_{z}=\bigvee\left\{w \in \mathscr{M}^{\mathrm{pr}}\right.$ : $z w=v z w\}$. Тогда $e-v_{z} \lesssim e-v$ и $v_{z}-$ наибольший из проекторов $w$, обладающих свойством $z w=v z w$. При этом $v_{z^{*}} z=v_{z^{*}} z v\left[4\right.$, c. 105]. Положим $r=p \wedge q \wedge q_{y} \wedge p_{x^{*}}$. Тогда

$$
\begin{aligned}
\tau(e-r) & =\tau\left((e-p) \vee(e-q) \vee\left(e-q_{y}\right) \vee\left(e-p_{x^{*}}\right)\right) \\
& \leqslant \tau(e-p)+\tau(e-q)+\tau\left(e-q_{y}\right)+\tau\left(e-p_{x^{*}}\right) \leqslant \delta+\beta+\beta+\delta=2 \delta+2 \beta .
\end{aligned}
$$

Поскольку $r \cdot x y \cdot r=r p_{x^{*}} \cdot x y \cdot q_{y} r=r \cdot p_{x^{*}} x \cdot y q_{y} \cdot r=r \cdot p_{x^{*}} x p \cdot q y q_{y} \cdot r=r p_{x^{*}} \cdot x p q y \cdot$ $q_{y} r=r \cdot x p q y \cdot r=r p \cdot x p \cdot q y \cdot q r=r \cdot p x p \cdot q y q \cdot r$, имеем $\|r \cdot x y \cdot r\| \leqslant\|p x p\| \cdot\|q y q\| \leqslant \varepsilon \alpha$. Лемма доказана.

Выбирая $V_{\varepsilon, \delta}$ с рациональными $\varepsilon, \delta>0$, из леммы 4 и теоремы Какутани получаем, что согласованная со структурой кольца на $\widetilde{\mathscr{M}}$ топология $d_{\tau}$ метризуема. Поскольку $U_{\varepsilon, \delta} \subset V_{\varepsilon, \delta}$ для всех $\varepsilon, \delta>0$, имеем $d_{\tau} \subset t_{\tau}$. Теорема 2 дает

СлЕДСТВИЕ $2\left[17\right.$, теорема 3]. Имеем $d_{\tau}=t_{\tau}$.

Автор выражает свою признательность участникам Казанского семинара “Алгебры операторов и их приложения" за полезные обсуждения.

\section{СПИСОК ЦИТИРОВАННОЙ ЛИТЕРАТУРЫ}

[1] Трунов Н. В., Шерстнев А. Н. Введение в теорию некоммутативного интегрирования // Итоги науки и техники. Современные проблемы математики. Новейшие достижения. Т. 27. М.: ВИНИТИ, 1985. С. 167-190.

[2] Kosaki H. On the continuity of the map $\varphi \mapsto|\varphi|$ from the predual of $W^{*}$-algebra // J. Funct. Anal. 1984. V. 59. № 1. P. 123-131.

[3] Kakutani S. Über die Metrization der topologischen Gruppen // Proc. Imp. Acad. Japan. 1936. V. 12. № 4. P. 82-84. 
[4] Nelson E. Notes on non-commutative integration // J. Funct. Anal. 1974. V. 15. № 2. P. 103-116.

[5] Бикчентаев А. М. F-нормированные идеальные пространства измеримых операторов. Казань: Казан. ун-т, 1988. (Рукопись деп. в ВИНИТИ 4 июля 1988 г., № 5375-В88 Деп.).

[6] Yeadon F. J. Non-commutative $L^{p}$-spaces // Math. Proc. Cambridge Phil. Soc. 1975. V. 77. № 1. P. 91-102.

[7] Bikchentaev A. M. On noncommutative function spaces // Amer. Math. Soc. Transl. (2). 1992. V. 154. P. $179-187$.

[8] Овчинников В. И. Симметричные пространства измеримых операторов // Докл. АН CCCP. 1970. T. 191. № 4. C. 769-771.

[9] Ciach L. J. Linear-topological spaces of operators affiliated with a von Neumann algebra // Bull. Acad. Pol. Sci. Math. 1983. V. 31. № 3-4. P. 161-166.

[10] Tikhonov O. E. On the Losanovskil class of condensing operators and its applications to non-commutative integration // Israel Math. Conf. Proc. 1999. V. 13. P. 204-208.

[11] Takesaki M. Theory of Operator Algebras. V. I. New York-Heidelberg-Berlin: Springer-Verlag, 1979.

[12] Szeptycki P. On solid spaces of measurable functions // Bull. Acad. Pol. Sci. Math. 1982. V. 30. № 3-4. P. 115-116.

[13] Kaplansky I. Projections in Banach algebras // Ann. Math. 1951. V. 53. № 2. P. 235-249.

[14] Peck N. T. On nonlocally convex spaces. II // Math. Ann. 1968. V. 178. № 3. P. 209-218.

[15] Yeadon F. J. Convergence of measurable operators // Proc. Cambridge Phil. Soc. 1973. V. 74. № 2. P. 257-268.

[16] Wnuk W. On a continuous embedding in a space of measurable functions // Bull. Acad. Pol. Sci. Math. 1986. V. 34. № 7-8. P. 413-416.

[17] Муратов М. А. Сходимости в кольце измеримых операторов // Сбор. науч. тр. Ташкент. ун-та. № 573. Функцион. анализ. Ташкент: Изд-во ТашГУ, 1978. Р. 51-58. 